\title{
Author Index for Volume IIO
}

Aaltonen, LA 2246

Aaronson, NK 1081

Abdel-Fatah, TMA 2450

Abderrahim-Ferkoune, A 2728

Abe, SK 1316

Abela, L 636

Abend, M 2738

Abnet, CC 1328

Abramson, DH 2623

Absenger, G 183, 435

Ackermann, I 2914

Adams, ME 706

Adams, RA 2178

Adenis, A 1148

Adjei, AA 320, 602

Adolfsson, J 1378

Affen, J 1681

Agarwal, D 1088

Agarwal, N 1917

Agger, WA 146

Aglietta, M 2165

Agorreta, J 1545

Agoulnik, S 1497

Aguilera, O 2700

Ahearn, T 1525

Ahmad, A 1211

Ahmed, N 133

Ahn, H 2472

Ahn, J-H 2472

Ahn, JS 384

Ahn, SD 1420

Ahn, Y-H 71

Ahonen, I 2905

Ahrens, M 1155

Ah-See, M-L 2847

Ainsworth, P 530

Airley, RE 715

Aissa, AB 2655

Aitken, Z 1908

Akakura, K 2389

Akanuma, N 189

Akashi, Y 1481

Akiyama, Y 1014

Akslen, LA 107

Akutsu, Y 189

Al Omran, R 1179

Alba, E 1139

Albanell, J 1139

Albiges, L 1778

Albiter, M 1413

Ali, R 224, 1322

Alias, Z 2187

Alimohammadi, A 984

Allan, S 1221, 1898

Allevi, G 2209

Allgood, PC 1834

Almstrup, K 668

Alonso, MR 1088

Alsner, J 2072

Al-Tameemi, W 715

Altman, DG 230

AlZoughbi, W 183

Amaral, AFS 2123

Amaro, A 1058

Amin, V 2847

Amofah, E 1221

An, H 2261

Andersen, JJ 146

Anderson, KE 2348

Anderson, LA 2796

Andersson, S 1561

André, T 2728

Andreu, M 1334

Andrews, J 2000

Andrews, PW 2131

Andrulis, IL 1367
Angelini, G 1058

Antoniou, AC 535, 1663

Aoe, K 2232

Aoki, D 2965

Aoyama, T 546, 2881

Apicella, C 1367

Appleton, K 1923

Appleyard, MV 2063

Aprile, G 2165

Arai, T 546

Araki, T 2923

Ardekani, GS 399

Ardine, M 2209

Aresu, M 256

Ariffin, H 1673

Arizumi, T 1943

Armel, S 530

Arminger, F 435

Arndt, GM 1189

Arns, M 984

Arora, S 2000

Asamura, H 1571

Asao, T 1985

Ascierto, PA 1721

Ashfield, A 565

Ashworth, A 1088, 1898

Assenat, E 1148

Assi, LK 2277

Assiotis, I 1663

Atias, D 2269

Atman, AA 2796

Atrih, A 1622

Attard, G 267

Aure, MR 2072

Ausems, MGEM 1081

Avan, A 172

Avery, KN 2829

Avivi, C 2269

Aziz, T 2593

Azzarone, B 1778

Baba, H 958

Baba, RA 706

Bachem, MG 409

Bader, FG 2081, 2544

Bae, DS 278

Bae, S 946

Bagnoli, M 330, 625

Baillie, GS 1278

Bain, GH 1525

Baker, SD 894

Balaguer, F 1334

Baldewijns, MMLL 797

Baldwin, DR 908

Baldwin, P 2837

Balermpas, P 501, 547

Balfoort-van der Meij, GAMA 1579

Balgkouranidou, I 2054

Balkwill, A 224, 2321

Ball, GR 1688, 2450

Ballester, M 2640

Bamber, JC 1727

Bamias, A 1917

Bandelloni, R 1058

Bang, Y-J 967

Bankart, JG 1101

Barbera, MA 2165

Barberis, M 1244

Barnadas, A 1139

Barnes, I 1322

Barrett-Lee, P 2139

Barrios, CH 1125

Barrowdale, D 1663

Barshack, I 2269

Barton, G 1622

Barton, P 2837
Bartsch, K 1427

Basso, G 1228

Bateson, M 928

Batty, GD 1820

Bauer, J 1342

Bauer, R 286

Bauer, S 1155

Bauernhofer, T 1614

Baulies, S 2195

Baumgartner, M 636

Baussano, I 7

Bævre, MS 2159

Bechmann, I 479

Beck, JF 656

Becker, K 1712

Becker, K-F 208

Becker, S 656

Bedognetti, D 2955

Beesley, AH 1189

Beham-Schmid, C 369

Behrens, C 1545

Behrens, G 1862

Beijnen, JH 2441, 2669

Belli, C 2165

Belloni, E 1244

Belousov, A 122

Benard, A 2935

Benchimol, D 2728

Bendell, JC 875, 2647

Bendifallah, S 2640

Benjamin, IJ 71

Benner, A 441

Bennouna, J 1148

Bensalah, K 1778

Ben-Shlomo, Y 2829

Beppu, T 958

Berahovich, RD 1179

Beral, V 224, 1322, 2321

Beresford, M 2847

Bergamo, F 2165

Berger, R 2269

Berger, W 984

Berhane, S 2090

Berkhof, J 1579

Berney, DM 616, 1655

Berry, P 1977

Bertan, C 1001

Bertheau, P 1413

Bertoni, R 2209

Bessa, X 1334

Bessen, T 859

Beswick, EJ 1211

Bezpalko, O 2116

Bhardwaj, A 2000

Bhargava, DK 2144

Bharwani, N 616

Bhat, HF 706

Bhoo-Pathy, N 2187

Bhuiya, T 976

Bi, L 842

Bian, SB 2011

Bianchi, P 1228

Bianchini, D 267

Bianco, R 2887

Biankin, AV 313

Biasiolo, A 2708

Bibeau, F 1148

Bichsel-Naef, M 2914

Biernat, W 991

Bighin, C 1133

Binysh, K 560

Birke, $\mathrm{H} 1841$

Bisagni, G 1133

Biswas, G 2144

Biswas, S 2144

Bjarnason, GA 648, 1250, 1917

Bjørge, L 107

Blank, S 1712

Blanke, CD 882

Blanks, R 560

Bleeker, MCG 1579

Bleiker, EMA 1081

Bliss, JM 256

Bock, E 1155

Boddy, AV 1977

Bodegas, ME 764

Bodmer, A 2655

Boerma, A 1535

Boers-Sonderen, MJ 888

Boezen, HM 1535

Boggi, U 172

Böhling, T 1446

Boige, V 2728

Boissière, F 1148

Bolla, MK 1088

Bollet, M 2195

Bonal, DM 2945

Bonas, SA 1101

Bonnetaud, C 1236

Bonzi, MC 2865

Booth, CM 551

Bordihn, K 286

Borgen, E 2072

Borgstein, PJ 1081

Borley, A 2139

Borner, M 1008

Boro, A 636

Boronina, TN 71

Borowiack, E 2837

Borrero, A 2700

Børresen-Dale, A-L 2072

Bottini, A 2209

Bottomley, A 2427

Bottomley, D 546

Boubli, L 1045

Bouchardy, C 788

Bouché, O 1148

Boudreau, HE 2569

Bouhidel, F 1413

Boult, JKR 1727

Bouma, WH 1081

Bourke, L 831

Bouzidi, MS 2615

Bowen, DT 1338

Bown, SG 1698

Boyer, J 1236

Boyer, MJ 2462

Boylan, M 2063

Bradbury, KE 232

Braganza, MZ 242

Brage, SE 2489

Brahmer, JR 602

Brain, KE 12

Brandi, G 2165

Bray, SE 1622 
Brugnoli, G 2209

Brundage, RC 304

Brunelli, M 2139

Brunetti, AE 2165

Bruno, M 2378

Bryan, RT 679

Bubendorf, L 2914

Bucci, G 1244

Büchler, MW 1712

Buchroithner, J 286

Budman, D 976

Buehler, S 1359

Buettner, R 549

Buffa, FM 715

Buffart, TE 546

Bujanda, L 1334

Bukowski, RM 1125

Bunce, C 1506

Bunch, KJ 1402

Bundred, N 573

Burggraaf, J 265

Burgués, O 1139

Burke, DJ 2569

Burris III, HA 875, 2647

Butler, AM 2369

Butler, R 2178

Butterworth, J 928

Buys, SS 1074

Büyükünal, E 1968, 2996

Byrne, A 1278

Byrne, AT 1307

Caballero, R 1139

Cahoon, EK 1825

Cai, AZ 2011

Cai, J 1199

Cai, Y 430, 1958

Calabrò, L 1721

Calin, GA 1614

Calvo, A 764

Calvo, L 1139

Cámara, MC 1139

Campbell, PT 1359

Caneppele, M 1053

Canese, R 625

Canevari, S 330, 625

Canlorbe, G 2640

Cao, B 63

Cao, J-Z 1767

Cao, Q 341

Cao, S 1733

Cao, SY 817

Cappelletti, MR 2209

Caramés, C 2700

Carballo, F 1334

Carcopino, X 1045

Carmassi, M 1045

Carnevale Schianca, F 1721

Carrasco, E 1139

Carrato, A 2123

Carter, B 584

Carton, M 1385

Cartwright, T 1438

Carvalho, B 546

Casado, V 2700

Cash, SE 146

Cassidy, J 1438

Castanon, A 1841

Castells, A 1334

Casterline, BW 2569

Castillo, L 2462

Castillo-Martin, M 2945

Catania, C 1244

Caval, V 2615

Cecconi, M 1058

Celesti, G 1228

Cénée, S 1385

Cereda, S 2165

Cerio, R 520

Chambers, PA 546

Chamizo, C 2700

Chan, A 1307

Chan, SYT 2450
Chand, M 19

Chang, DK 313

Chang, G 916

Chang, H 2224

Chang, L 353

Chang, PM-H 208

Chang, W 384

Chanock, S 2123

Chantrill, LA 313

Charles, AK 1189

Charpin, C 1045

Chau, C 1759

Chau, I 19

Chazal, M 2728

Chen, B 2109

Chen, C 741, 1288, 1785, 1958

Chen, C-Y 49

Chen, E-Z 1871

Chen, G 63, 859, 2224

Chen, GG 1066

Chen, $\mathrm{H} 602$

Chen, K 341

Chen, K-W 208

Chen, L 1014, 1465, 2011, 2261, 2514

Chen, M 2300, 2396

Chen, M-H 2224

Chen, P 1871, 2327

Chen, Q 802

Chen, S 1288, 1958

Chen, S-Y 1552

Chen, W 450, 695, 1767

Chen, X 946

Chen, Y 1014, 1351, 2224

Chen, Y-m 808

Chen, Z-H 1767

Chen, Z-Y 733

Cheng, A-L 2434

Cheng, KK 679

Cheng, S 1811

Cheng, YY 510

Chernikova, SB 1179

Chiam, K 2462

Chiarion-Sileni, V 1721

Chie, EK 1420

Chikamatsu, K 2506

Chikamoto, A 958

Chimonidou, M 2054

Chin, VT 313

Cho, CH 278

Cho, E 249

Choi, BS 1221

Choi, DH 1420

Choi, MK 384, 2472

Choi, YL 384

Chondrou, PS 2217

Chong, MG 1506

Chornokur, G 530

Chou, A 313

Chouaib, S 1778

Choueiri, TK 1433, 1917

Chowdhury, S 616

Choy, E 2896

Christen, T 2935

Chu, E 1438

Chu, R 2327

Chu, X 2812

Chu, Y 353

Chuan-Hao Kao, S 510

Chung, HH 1878

Chung, J 1634

Chung, J-H 2688

Chung, JM 1634

Cihoric, N 2914

Cillo, U 2708

Cirillo, M 1133

Cirkel, GA 2441

Cisar, L 2821

Ciuleanu, T 55

Civini, S 2955

Clamp, AR 1118

Clark, D 1342

Clark, JI 2821

Clark, JP 1655
Clark, SJ 2462

Clarke, NW 2099

Clements, D 83

Clements, M 1378

Clinch, N 2159

Coates, PJ 2063

Cobo, M 55

Coens, C 2427

Coldman, AJ 556

Cole, CH 1189

Cole, PA 71

Cole, RN 71

Coleman, J 2420

Collie-Duguid, E 1525

Colomban, O 1517

Colvin, EK 313

Colzani, E 1378

Cong, YJ 817

Conrads, KA 123

Conrads, TP 123

Conroy, T 1148

Cooper, CS 1655

Copson, E 230

Corbelli, J 2165

Corcoran, A 899

Cordon-Cardo, C 2945

Corson, JM 2479

Cortez, A 2640

Cosme, A 1334

Costa, C 2662

Cote, G 2896

Cottu, P 2195

Cottu, P-H 1413

Coussy, F 1413

Coutant, C 2640

Covens, A 609

Coviello, DA 1058

Cox, HJ 489

Cox, T 2090

Crabb, SJ 1759

Cramer, DW 1392

Craparotta, I 2865

Crapez, E 1148

Creutzberg, CL 868

Crippa, F 26

Cristóbal, I 2700

Croner, RS 2544

Cubie, H 2804

Cubiella, J 1334

Cui, A 1595

Cui, JX 2011

Cui, L 450, 1595

Cui, W 341

Cumming, P 560

Cummings, C 1727, 2369

Cunningham, AP 535

Curtin, NJ 1977

Cuschieri, K 2804

Cutress, RI 230

Cutz, J-C 2593

Cuvier, C 1413

Cuzick, J 827, 1655, 1681

Cyr, D 1385

Czapiewski, P 991

Czene, K 1378, 1908

Dadaev, T 1663

Daemen, T 1535

Dahiya, R 1645

Dahle-Smith, A 1525

Dai, J 2291

Dai, N 2291

Dai, W 1785

Daidone, MG 26

Daigeler, A 1456

Dalianis, T 1793

Dallaglio, K 199

Dallal, CM 2339

Dalpiaz, O 2531

Daly, MB 1074

Daly, RJ 2462

Danforth, KN 2339

Dang, NH 2232

Daniëls, LA 868

Danielsen, HE 2159

Daraï, E 2640

Darcy, KM 123

Das, K 546

Datta, PK 946

Dattola, F 1228

Daveau, C 2195

Davies, C 2829

Davies, N 1506

Davis, M 2829

Dawe, S 1681

Dawson, H 1008

Dawson, SN 2405

Dayalan Naidu, S 71

D'Amato, C 2887

D'Amato, V 2887

de Aberasturi, AL 764

de Beer, JC 2361

de Bono, JS 267

De Braud, FG 26

de Bruijn, P 888

de Bruijn, P 894

De Cecco, L 330

De Galitiis, F 1721

De Giorgi, V 2955

de González, AB 242

de Jonge, MJA 2441

de la Haba, J 1139

de Leeuw, JRJ 593

de Liaño, AG 2201

De Melo, J 2593

De Pas, T 1244

De Placido, S 2887

de Roos, A 265

de Roos, MA 1081

de Roquancourt, A 1413

de Thé, H 1413

de Vathaire, F 2623

Dearnaley, D 267

Dearnaley, DP 256

Deb, S 1606

Debbage, P 2677 
Dietrich, LL 146

Dietrich, P-Y 2655

Dietz, A 479

Dileo, P 2420

Dilks, P 616

Din, NU 584

Dina, R 115

Ding, X-W 2378

Ding, Y 1288, 2291

Dinkova-Kostova, AT 71

Dirix, LY 375

Dite, GS 1367

Dixit, A 2144

Do, I-g 384

Doki, Y 164, 1163

Dome, B 984

Donaghy, M 2804

Dong, J 2560

Dong, W 842

Dong, Z 1801

Donnelly, C 12

Donnelly, LS 1681

Dono, M 1058

Donovan, JL 2829

Donskov, F 1917

Dos Santos, L 976

dos-Santos-Silva, I 1908

Douglas, J 1759

Douillard, J-Y 55

Dowlati, A 2647

Down, L 2829

Downey, CL 1744

Dowsett, M 1898, 2847

Drake, T 1759

Drayson, M 1506

Drecoll, E 2081

Drew, PA 775

Du, J 2291, 2310

Du, L 1801

Du, P 450

Du, X 63

Duan, Z 2896

Dubé, V 609

Dubuc, AM 636

Duckworth, CA 741

Dudás, J 2677

Dudek, AZ 304

Duffy, SW 560, 1834

Dulla, K 1278

Dunkel, N 2655

Dunn, J 1950

Durcan, L 230

Durrani, FA 1733

Dutton, P 2139

Easton, DF 535

Ebsworth, K 1179

Eccles, B 230

Edgar, AB 1342

Ediebah, DE 2427

Edin, S 2551

Eeles, R 1663

Eguchi, H 164

Eichberger, P 2677

Eichholz, A 1655

Eilers, G 2479

Eisen, A 530

Eliott, J 859

El-Khoueiry, AB 882

Ellis, IO 1688, 2450

Elovainio, M 1820

Endersby, R 1189

Engels, EA 2796

Enomoto, T 1481

Escudier, B 1125, 1778, 2821

Eskens, FALM 888, 2170

Espeli, V 2655

Espié, M 1413

Esposito, AI 1058

Etienne-Grimaldi, M-C 2728

Etuk, A 1221

Evans, AR 715

Evans, DG 827, 1681
Evans, S 2829

Extra, J-M 1413

Fabri, LJ 2855

Failes, TW 1189

Falcone, A 2165

Falk, C 441

Fan, P 1488

Fan, S 695, 1767

Fan, X 842

Fan, Y-y 808

Fang, J 1014

Fang, L 430

Fang, Y 1767

Farè, E 26

Farmer, R 1506

Farzaneh, F 1221

Fasola, G 2165

Fasolato, S 2708

Faucheron, J-L 2728

Feichtenschlager, V 1427

Feichtinger, J 286

Feith, M 2985

Felley-Bosco, E 2040

Feltbower, RG 1338

Feng, Y 2300

Feng, Y-M 724

Fenwick, K 1663

Fernández, AF 2123

Ferraldeschi, R 267

Ferrari, E 1189

Ferrero, G 2209

Ferris, JS 1074

Ferrozzi, F 2209

Ferrua, B 1236

Ferrucci, PF 172

Fiaschetti, G 636

Field, JK 1834

Figlin, RA 1125

Filipits, M 984

Filippi, R 2165

Fina, F 1045

Finegan, M 1525

Fini, L 1228

Finlayson, A 1322

Fisher, D 2178

Fisher, G 1655

Fisher, J 304

Flechtner, H 2427

Fleming, I 1525

Fleming, S 1622

Fletcher, JA 2479

Fly, K 1125

Fodstad, Ø 2072

Fokas, E 501, 547

Folkerd, EJ 1898

Fontaine, H 2170

Forbes, BE 2855

Forbes, LJL 12

Formento, J-L 2728

Formento, P 2728

Formisano, L 2887

Fornaro, L 2165

Fossa, SD 256

Foster, CS 1655

Foukakis, T 1378

Foulkes, WD 530

Fox, J 1681

Fox, R 2090

Fox, SB 1606

Fraefel, C 94

Fraga, MF 2123

Franceschi, S 7

Francois, E 1148

Fraser, FW 133

Freedman, ND 242, 1328, 2339

Freyer, G 1517

Fritsch, H 2434

Fry, DJ 1118

Fu, B 741

$\mathrm{Fu}, \mathrm{J} 450$

Fu, L 1014

Fu, Q 146
Fu, S 1801

Fujii, T 2965

Fumagalli, C 1244

Funahashi, Y 1497

Funel, N 172

Furber, L 1101

Furniss, DL 2178

Furukawa, H 1163

Furuta, K 1571, 2716

Furuya, K 2881

Fusciello, C 2887

Gabra, H 1923

Gadellaa-van Hooijdonk, CGM 2441

Gajda, M 2537

Gajjar, KB 2874

Galais, MP 1148

Gale, CP 1338

Gameiro, SR 1472

Gamoh, M 1163

Gan, Y 817

Ganesan, R 2837

Gao, B 602

Gao, H 1552

Gao, J 450

Gao, K 2310

Garcia, S 1045

García-Closas, M 2123, 2412

García-Closas, R 2123

García-Foncillas, J 2700

Garibaldi, JM 1688

Gariboldi, M 1001

Gariboldi, MB 2865

Gathani, T 224

Gatta, A 2708

Gattani, S 510

Gatterbauer, B 286

Gaub, M-P 2728

Gavine, P 1169

Gavine, PR 967

Ge, D 353

Geldart, T 1759

Generali, D 2209

Geng, H-Z 1748

Genkinger, JM 1074

George, J 1606

Georgoulias, V 2054

Geppert, C-I 2544, 2985

Gerdes, C 1221

Gerestein, CG 42

Gerger, A 183, 369, 435, 1614, 2524

Gerlach, MM 479

Gerty, S 230

Ghadirian, P 530

Ghanim, B 984

Ghilotti, M 1001

Ghorab, Z 609

Giacchetti, S 1413

Giannatempo, P 26

Gianni, AM 26

Giatromanolaki, A 2209, 2217

Gierach, GL 2339

Gilbert, FJ 1525

Giles, GG 1367

Gill, AJ 313

Gillams, A 1698

Gilly, F-N 2728

Gimenez-Capitan, A 2662

Giovannetti, E 172

Giovannucci, EL 249

Giraudi, S 1133

Glaser, A 1338

Glass, DC 783

Glasspool, RM 1923

Gnanapragasam, VJ 2405

Gnoni, R 1133

Goertz, O 1456

Goh, C 1663

Golan, T 2269

Goldbohm, RA 797

Goldstein, RS 2269

Golovine, K 899

Gong, I-Y 882

González-Neira, A 1088

Gooden, MJM 1535

Goossens-Beumer, IJ 2935

Gore, ME 1125, 1923

Gorman, P 1053

Goryca, K 991

Gotay, C 2427

Goto, T 2881

Govindasami, K 1663

Grabellus, F 1155

Grabsch, HI 546, 967, 1525

Graesslin, O 2640

Granata, A 330

Granchi, C 172

Green, AR 1688

Green, J 224, 2321

Green, R 1359

Greenhalgh, R 1681

Greeno, EW 304

Gregersen, PK 976

Greimel, E 2427

Gridley, G 1825

Griggs, K 510

Grimm, M-O 2537

Grisold, W 286

Grizzi, F 1228

Grizzle, WE 2000

Grønhøj Larsen, C 1587

Gronwald, J 530

Grotzer, MA 636

Gruber-Mösenbacher, U 286

Grunder, E 636

Grüner, R 107

Grunewald, S 1155

Grusch, M 984

$\mathrm{Gu}, \mathrm{A} 1871$

$\mathrm{Gu}, \mathrm{J} 353$

$\mathrm{Gu}, \mathrm{Y} 1169$

Gualco, M 1058

Guan, W 2662

Guan, Y 297

Gugger, M 2914

Guida, F 1385

Gujar, SA 83 
Hamaguchi, T 2716

Hamberg, P 888

Hamdy, FC 2829

Hamilton, CA 123

Hamilton, W 584

Hamoudi, R 520

Hampton, C 489

Hamy, A-S 1413

Han, F 49, 297

Han, J 842, 1855, 2291

Han, K 63

Han, S 2560

Han, W 1351

Hanby, A 1053

Hanby, AM 1744

Hansson, J 2489

Hara, M 469

Harati, K 1456

Harbison, C 648

Hardisson, D 1088

Hardy, MP 2855

Hargest, R 421

Hariharan, S 2821

Harker, D 560

Harmon, D 2896

Harris, AL 715, 2209

Harris, B 715

Harris, S 489

Harrison, DJ 2975

Harshman, LC 1917

Hart, CA 2099

Hartman, M 2187

Hartmann, A 2985

Harwood CA 520

Hasan, J 1118

Hasan, T 1698

Hashimoto, D 958

Hashimoto, S 1481

Hasmim, M 1778

Hatano, R 2232

Haustein, V 753

Hawkins, MM 2623

Hawkins, NJ 1606

Hayashi, H 958

Hayashi, T 156

Haybaeck, J 286

Hayden, R 1506

He, H 1785, 2021

He, J 2812

$\mathrm{He}, \mathrm{K} 1847$

He, M 392

Head, J 1759

Hedman, H 1793

Hegedus, B 984

Heideman, DAM 546

Heikenwälder, M 686

Heinonen, H-R 2246

Hellman, K 1561

Helpman, L 609

Helson, E 83

Henderson, DJP 1278

Hendrikx, JJMA 2669

Hendryx, M 1847

Heng, DYC 1433, 1917

Hénin, E 1517

Henriksson, R 1793

Henry, M 2615

Hernandez, V 1334

Herranz, J 1088

Hibshoosh, H 1367

Higuchi, T 1985

Hilger, RA 1155

Hill, EM 2829

Hinoda, Y 1645

Hintsa, T 1820

Hirakawa, M 156

Hirano, K 1943

Hirata, H 1645

Hironaka, S 27

Hirsch, T 1456

Hisada, T 2047

Hitrata, J 2881

Ho Kim, W 967
Ho, RLK 1066

Hoda, MA 984

Hodge, JW 1472

Hodgkinson, E 2178

Hoefler, G 183, 1614

Hoffmann, R 1278

Höfler, H 2081

Hofman, P 1236

Hofman, V 1236

Hogervorst, FBL 1081

Hoh, CK 875

Hoiczyk, M 1155

Holdgaard, PC 363

Hollema, H 1535

Hollenbeck, AR 242, 1328, 2339

Holliday, DL 1744

Homer, KE 831

Honeywell, R 172

Hong, B-S 2472

Hong, JH 2472

Hong, S-H 1298

Hong, S-W 2472

Honma, Y 2716

Hood, BL 123

Hopkins, L 1342

Hopper, JL 1367

Hordijk, G-J 593

Horie, S 2583

Hornicek, FJ 2896

Horvath, LG 313, 2462

Horwich, A 256

Hoshida, Y 2708

Hoshino, I 189

Hosokawa, A 271

Hospitel, M 2170

Hostomsky, Z 1977

Hottinger, AF 2655

Hou, L 1351

Hou, T 1260

Hou, W 1992

Hou, Y 341

Houry, S 2728

Houslay, MD 1278

Howell, A 1681

Howlett, M 1189

Hsu, C-H 2434

$\mathrm{Hu}, \mathrm{B}-\mathrm{g} 1066$

$\mathrm{Hu}, \mathrm{G} 2310$

$\mathrm{Hu}, \mathrm{S} 894$

$\mathrm{Hu}, \mathrm{X} 189$

$\mathrm{Hu}, \mathrm{Z} 2291$

Huang, C 2812

Huang, DC-L 2434

Huang, F 1199

Huang, H 842

Huang, J 842, 1288, 1958

Huang, J-M 1748

Huang, M 430

Huang, R 2250

Huang, S 450

Huang, S-L 2378

Huang, W 2300

Huang, W-Y 2354

Huang, X 1034, 1260

Huang, Y 1260, 2560

Huang, Y 430

Huang, Z 695

Hubbard, RB 908

Huddart, R 256

Hudis, CA 829

Hudry, D 2640

Huggett, MT 1698

Hughes, LJ 2829

Hughes, RG 2277

Huguet, F 2640

Huh, SJ 1420

Hui, T 1269

Huitema, ADR 244

Humphreys, K 1908

Humphris, JL 313

Hundsberger, T 2655

Hunter, DJ 1855

Hunter, JE 928
Husby, JA 107

Hutson, TE 1125, 2821

Hutterer, GC 2531

Hveem, TS 2159

Hyde, SJ 2000

Hylander, BL 320

Iacovelli, R 1778

Ibrahim, RI 2187

Ichikawa, D 271

Ide, S 2923

Ilie, M 1236

Illing, R 1698

Illvan, S 1968, 2996

Im, S-A 967

Im, Y-H 384

Imai, H 2047

Imai, K 958

Imamura, H 1163

Inam, I 546

Inderbitzin, D 1008

Infante, JR 875, 2647

Ingebrigtsen, VA 2072

Ingold, B 686

Inoue, M 1316

Inoue, Y 2923

Inskip, PD 242, 1825

IntHout, J 42

Iorio, E 330, 625

Iovanna, J 1045

Iqbal, J 530

Iqbal, S 882

Isayama, H 1943

Ishiguro, A 271

Ishihara, J 1316

Ishiko, T 958

Ishimoto, T 958

Ishioka, NS 1985, 2506

Ismail, IA 1298

Ismiil, N 609

Iso, H 792

Isozaki, Y 189

Ito, $\mathrm{H} 1088$

Ito, M 1027

Ito, Y 1943

Ivan, C 1614

Iwasa, S 2716

Iwasaki, M 1316, 2765

Iwata, H 1088

Iwata, S 2232

Iwata, T 2965

Iwaya, K 2881

yama, S 156

Izumi, H 2583

Jackson, BP 2123

Jaen, JC 1179

Jahn, SW 1614

Jakobsen, A 363

Jakopovic, M 984

James, ND 679

Jamieson, GG 775

Jamin, Y 1727

Jänicke, F 753

Janssen, K-P 2081

Januszewski, A 4

Jasani, B 2139, 2178

Jassem, E 991

Jassem, J 991

Jayson, GC 1118

Je, Y 2772

Jen, Y-M 2354

Jenkins, J 560

Jensen, DH 1587

Jenster, G 1278

Jeong, I-G 2472

Jermyn, M 1698

Jheon, S 2688

Ji, A 2514

Ji, J 421

Ji, K 421

Jia, AY 2945

Jia, H-L 1748

Jia, W-H 2633

Jia, Y 421

Jiang, C 842

Jiang, G 2496

Jiang, J 1351

Jiang, P 353

Jiang, T 2560

Jiang, WG 421

Jiang, Z-Y 1871

Jimenez, S 1936

Jin, G 2291

Jin, J 2955

Jin, P 2955

Jin, Y 2688

Jin, Z 2514

Jochem, C 1862

Joensen, UN 2604

Joensuu, H 1446

Jogai, S 489

Johansson, ALV 1378

Johansson, CH 2489

John, EM 1367

Johns, AL 313

Johnson, C 2450

Johnson, PJ 2090, 2277

Jokela, M 1820

Jones, C 1977

Jones, JL 1744

Jones, L 230, 829

Jones, M 1088

Jones, ME 1898

Jones, SF 2647

Jones, TM 269

Jonker, DJ 648

Jonkers, J 2747

Jordan, LB 565, 1744

Jordan, VC 1488

Jørgensen, A 668, 2604

Jotte, RM 602

Jover, R 1334

Jugurnauth-Little, S 1663

Jung, E-J 967 
Kayaleh, OR 882 Kaye, S 1923

Ke, D 1034

Ke, J 430

Ke, T 1034

Keefe, D 859

Keegan, TJ 1402

Kees, UR 1189

Keijzer, R 2935

Keimling, M 1862

Keller, G 2081

Kench, JG 313

Kennedy, CR 146

Kenny, SL 2178

Kent, E 1698

Keogh, M 976

Keum, KC 1420

Key, TJ 2321

Khakwani, A 908

Khalifa, MA 609

Khalili, H 976

Kham, SKY 1673

Khambata-Ford, S 648

Khan, H 1211

Khan, K 2837

Khanday, FA 706

Khanim, F 1506

Khatri, A 304

Khaustova, N 753

Khosravi, S 399

Kiefer, A 286

Kiesslich, T 1614

Kii, T 27

Kilgour, E 967

Kilsdonk, MJ 850

Kim, BG 278

Kim, D-Y 34

Kim, H 2688

Kim, HS 1878

Kim, IY 1634

Kim, JH 1420

Kim, J-H 34

Kim, J-K 1298

Kim, JW 278

Kim, K 1420

Kim, K 2688

Kim, KT 278

Kim, MH 278

Kim, S 875, 2821

Kim, SM 278

Kim, S-O 34

Kim, SS 1420

Kim, TH 1634, 1878

Kim, W-J 1634

Kim, Y 2472, 2772

Kim, YB 278, 1420

Kim, Y-M 34

Kim, YT 278

Kim, Y-T 34

Kim-Sing, C 530

Kimura, T 1497, 2583

King, EV 489

King, MT 2427

Kioi, M 1179

Kirschner, MB 510

Kirstein, MN 304

Kishimoto, T 2232

Kiss, K 1587

Kitada, S 2583

Kitahara, CM 242

Kitajima, T 2923

Kivimäki, M 1820

Klarenbeek, S 2747

Klebe, S 510

Kleinerman, RA 2623

Klepetko, W 984

Klikovits, T 984

Kloth, JSL 2441

Klupp, F 441

Knight, JA 1367

Knox, JJ 1917

Ko, JJ 1917

Kobayashi, A 2965
Kobune, M 156

Koch, M 441

Koelzer, VH 1008

Kogevinas, M 2123

Kogure, H 1943

Kohno, K 2583

Kohno, T 1571

Kohut, K 1053

Koike, K 1943

Kolenko, VM 899

Koljonen, V 1446

Kollmannsberger, CK 1433

Komatsu, Y 1163

Komiya, E 2232

Kondo, C 271

Kondo, N 1088

Kondo, S 2923

Kong, X 1014

Konge, L 1587

Koole, R 593

Koort, HEK 1179

Kornprat, P 183

Koshiol, J 2796

Kote-Jarai, Z 1663

Koukourakis, MI 2209, 2217

Koussounadis, A 2975

Kovacs, G 1655

Kowalska, M 2837

Kozarewa, I 1663

Krämer, OH 656

Kratzke, RA 304

Kreiter, E 1891

Krenn-Pilko, S 2524

Kristensen, DG 668

Kroeger, N 1917

Krohn, M 2072

Krol, ADG 868

Kronqvist, P 2905

Kruitwagen, RFPM 42

Kuang, Y 2479

Kubick, C 479

Kubo, T 792

Kuchenbaecker, KB 535

Kuchiba, A 2765

Kuenen, MA 1081

Kuiper, R 2747

Kulka, J 1744

Kumada, T 2090

Kumar, J 133

Kumar, S 2116

Kunz, C 441

Kuo, CJ 602

Kuopio, T 2905

Kupets, R 609

Kuppen, PJK 459, 2935

Kurashige, J 164

Kurokawa, T 1481

Kurokawa, Y 1163

Kuroki, H 958

Kusama, R 2765

Kushima, R 1571

Kusunoki, M 2923

Kutikov, A 899

Kuwano, H 1985

Kuznetsov, G 1497

Kwilas, AR 1472

Kyle, S 1977

Lackner, C 183

Laden, F 1855

Lagas, JS 2669

Laghi, L 1228

Lai, PBS 1066

Lajud, SA 2116

Lalai, R 2747

Lalloum, M 2195

Lam, PYP 94

Lamb, HJ 265

Lamba, JK 304

Lambert, SR 520

Lamont, DJ 1622

Lanas, Á 1334

Landoni, E 26
Landy, R 1841

Lane, JA 2829

Lane, RSD 214

Langdon, SP 2975

Langer, C 648

Langer, R 1712, 2985

Langsenlehner, T 2524

Langsenlehner, U 2524

Lankheet, NAG 2441

Lanocita, R 26

Lanza, F 1058

Lardinois, D 2914

Larkin, JM 1125

Larzabal, L 764

Lasitschka, F 441

Lasser, P 2728

Laszlo, V 984

Lathia, JD 1179

Lau, WY 1110, 1811

Laurent-Puig, P 1148, 2728

Lauttia, S 1446

Lavelle, K 573

Lee, AJ 535

Lee, AT 976

Lee, BY 2462

Lee, C 278

Lee, CT 2688

Lee, DH 1634

Lee, GT 1634

Lee, H-J 1298

Lee, H-S 1420

Lee, J-L 1433, 1917, 2472

Lee, K-H 2472

Lee, NK 1420

Lee, PWK 83

Lee, S 1438

Lee, S-W 34

Lee, TS 278

Leeds, J 1525

Leek, R 715

Leffers, N 1535

Leffondré, K 1385

Lehmann-Che, J 1413

Lehnhardt, M 1456

Lehtiö, J 2489

Lei, T-C 2496

Leigh, I 520

Leighl, NB 602

Leitzmann, MF 1862

Leivonen, S-K 2072

Lemetre, C 1688

Leng, Y 450

Lenz, H-J 882

Leo, E 1001

Leon, LG 172

Leone, F 2165

Leongamornlert, D 1663

Leto, TL 2569

Letoublon, C 2728

Levaggi, A 1133

LeVea, C 320

Levinson, KT 2647

Li, B 1767

Li, C 1595, 2327

Li, D 2116

Li, E 2109

Li, F 1992

Li, G 392, 399

Li, H 1034, 2479

Li, J 63, 695, 1110, 1351, 1606, 1727, $1801,1908,2131,2327$

Li, JY 2011

Li, K 2396

Li, L 1465, 2300, 2633, 2812

Li, N 1811

Li, Q 916, 2560

Li, S 2812

Li, S-H 733

Li, SP 2847

Li, W 1801

Li, X 392, 802, 2327, 2479

Li, X-Q 724

Li, XR 2310

Li, Y 341

Li, Z 1801

Lian, J-J 1552

Liang, C-Z 1767

Liang, N 2812

Liang, S 976

Liang, Y-C 1748

Liang, Z 1595

Lianidou, ES 2054

Liao, B 1767

Liao, X-H 560

Liao, Y 1074, 1367, 2250

Liao, Y-B 1767

Liebenberg, L 2361

Liefers, GJ 459, 2935

Liest $\varnothing 1, \mathrm{~K} 2159$

Liliac, L 625

Liljegren, A 1378

Lim, HY 2821

Lim, T 546

Lin, A-H 1465, 2633

Lin, C-C 2434

Lin, CW 2310

Lin, C-Y 2354

Lin, H-C 1847

Lin, H-M 2462

Lin, HP 1673

Lin, J-C 2354

Lin, S 2785

Lin, T 842

Lin, X 1125, 2514

Lin, X-1 808

Lin, Y 916

Lin, Z 695, 2261

Lindblom, A 1088

Lindquist, D 1561, 1793

Ling, A 2551

Linton, A 510

Lintunen, M 2905

Lis, LG 304

Little, MP 2623

Liu, B 1351, 2662 
Lu, Y-k 808

Lu, Y-S 2434

Lu, ZX 817

Lubinski, J 530

Luce, D 1385

Lugli, A 1008

Lukas, P 2677

Lunardi, G 1133

Luo, H 392

Luo, J 1847

Luo, J-H 1767

Luo, L-Y 1785

Luo, T-C 1552

Lutrino, SE 2165

Lv, J 1169

Lv, L 2310

Lv, T 2261

Lynch, HT 530

Ma, G-F 1552

Ma, H 2291

Ma, J 1465, 2633

Ma, L 916

Ma, L-L 1552

Ma, WW 320

Ma, X 1014, 2250

Mach, N 2655

Mackean, M 1923

MacKenzie, MJ 1917

Madadi, AR 146

Madoz-Gúrpide, J 2700

Maftouh, M 172

Magidson, J 2756

Magnussen, IJ 107

Mahamat, A 2728

Mahner, S 753

Mahon, KL 2462

Mai, H-Q 2633

Maier, H 286, 2677

Maio, M 1721

Maiorana, A 199

Maishman, T 230

Mäkelä, R 2072

Makhov, P 899

Mäkinen, N 2246

Makris, A 2847

Malats, N 2123

Malesci, A 1228

Malhaire, C 2195

Malinowsky, K 2081

Mallaev, M 1008

Mallesara, G 2462

Mallon, P 2195

Malone, D 1221

Malone, DP 648

Maltseva, D 753

Małysiak, S 2837

Mancini, M 2865

Mandalà, M 1721

Mankin, H 2896

Mannweiler, S 2531

Mansutti, M 1133

Mao, X 63

Mao, Y-P 1465, 2633

Marcato, P 83

Marchetti, P 1721

Marciano, R 2887

Marconi, A 199

Marenco, D 1133

Margolin, S 1088

Marhold, F 286

Mariani, L 26

Maric, I 1058

Marincola, F 2955

Mariyannis, A 1785

Marosi, C 286

Maroto, JP 2201

Marquette, C-H 1236

Márquez, M 2123

Marshall, A 1950

Martell, B 1125

Martin, A 679

Martin, C 2201
Martin, FL 2874

Martín Hernández, JM 2531

Martin, L 1936

Martin, N 115

Martin, RM 2829

Martínez-Useros, J 2700

Martin-Hirsch, PL 2874

Martinka, M 399

Martinotti, M 2209

Marty, M 1413

Maruyama, K 2389

Maruyama, T 189

Marx, C 656

Marx, G 2462

Mason, C 976

Massa, J 249

Massuger, LFAG 42

Masui, O 1250

Matczak, E 1125

Mathijssen, RHJ 894, 2441

Matias-Guiu, X 2544

Matsubara, H 189

Matsui, H 1943

Matsui, J 1497

Matsumoto, S 1027

Matsumura, T 164

Matsuo, K 271, 1088

Matthes, S 2537

Matthies, C 2738

Maughan, TS 2178

Mauri, FA 208, 1930

Mavaddat, N 535

Mavropoulou, S 2217

Maxwell, GL 123

May, AM 593

Mayer, M 2756

Mazard, T 1148

McArdle, SEB 2450

McCarthy, F 1655

McCaughan, BC 510

McCormack, R 55

McCormack, V 1908

McCulloch, DR 133

McDermott, DF 1433

McGurk, A 1118

McHugh, P 715

McIver, B 1322

McKay, MJ 1606

McKenna, E 1438

Mckiddie, F 1525

Mclaughlin, JR 1359

McLaughlin, L 1221

McMillan, DC 1409

McNeil, K 2855

McNeish, IA 1923

McShane, CM 2796

McSorley, J 560

Mead, G 1759

Meade, AM 2178

Meads, C 2837

Meeran, K 115

Mehrazin, R 899

Mei, Z 1595

Meijer, CJLM 1579

Meijer, GA 546

Meineke, V 2738

Mellado, B 2201

Mellows, T 489

Melotti, F 1244

Mendiola, M 1088

Menéndez-Rodríguez, P 1088

Meng, F 2479

Menvielle, G 1385

Menzin, A 976

Mercer, T 2139

Merchant, M 1179

Merok, MA 2159

Merritt, MA 1392

Merson, S 1655

Merz, F 479

Messai, Y 1778

Messina, C 26

Metcalf, RL 1118
Metcalfe, C 2829

Metellus, I 1045

Meyer, KB 1088

Mezzanzanica, D 330, 625

Miao, L 94

Miao, Q 1552

Micetich, KC 882

Michaelson, MD 2821

Michailidou, K 1088

Michel, Y 501, 547

Migita, K 1027

Mikami, K 792

Miki, T 792

Milaki, G 2054

Milani, M 2209

Milano, G 2728

Milde-Langosch, K 753

Milella, M 2165

Milenkova, T 55

Miles, D 2847

Mima, K 958

Mimori, K 164

Min, K 1634

Minutolo, F 172

Miquel, ME 616

Mirisola, V 1058

Mishra, SK 2144

Missmer, SA 1392

Miwa, H 1163

Miyagi, Y 546

Miyamoto, M 2881

Miyamoto, R 1481

Miyanishi, K 156

Miyazaki, T 1985

Mladkova, N 520

Moch, H 686

Modarressi, A 788

Modiano, MR 602

Mohamed, Z 208

Mohamed-Bashir, SA 94

Mohri, Y 2923

Møller, H 1655

Moller, P 530

Monshi, B 1427

Monti, E 2865

Montorsi, M 1228

Montuenga, LM 764, 1545

Moon, KH 1634

Moore, MJ 648

Morandi, P 199

Moreno, LT 1088

Morgan, M 2139

Morgan, S 967

Mori, M 164, 792

Morillas, JD 1334

Morimoto, C 2232

Morosi, C 26

Morreau, H 2935

Morris, KT 1211

Morrison, L 2985

Mortensen, N 1606

Mosci, C 1058

Moseley, PM 2450

Moser, KA 224

Moss, S 1908

Moss, SM 2412

Mosse, S 1698

Motzer, RJ 1125, 2821

Mouchli, A 2116

Mouroux, J 1236

Mudaliar, MAV 1622

Muehr, M 2537

Mühlenberg, T 1155

Mukherii, D 267

Muley, T 441

Mulkearns-Hubert, EE 1179

Müller-Myhsok, B 2738

Mulligan, AM 1367

Muo, C-H 2354

Murakami, K 189

Murase, K 156

Muro, K 271

Murphy, MFG 1402

Murray, DW 1307

Murray, GI 1525

Murray, J 1977

Murray, K 2063

Murray, T 1221

Murtagh, GM 110

Nabi, G 1622

Nagamori, S 2030, 2506

Nagano, O 958

Nagarajah, J 1155

Nagasaki, T 469

Nagashima, T 2047

Nagda, DA 2116

Nagrial, AM 313

Nagy, E 2874

Nakagawa, S 958

Nakai, Y 1943

Nakajima, Y 1027

Nakamura, M 271

Nakanishi, H 469

Nam, BH 278

Nam, J-H 34, 278

Nanthakumaran, S 1525

Napoli, CU 686

Nappi, L 2887

Narod, SA 530

Nasca, PC 1351

Nasciuti, F 1058

Näsman, A 1793

Neal, DE 2405, 2829

Neal, JW 602

Neal, RD 584

Necchi, A 26

Nelson, A 2178

Nemoto, K 2389

Nenutil, R 2063

Nesbakken, A 2159

Nesland, JM 2072

Neuhausen, SL 530

Neumeister, P 369

Newman, JP 94

Newsted, D 1250 
Ogston, S 565

Ohara, Y 1481

Ohgaki, H 636

Ohkohchi, N 148

Ohnuma, K 2232

Ohshima, Y 1985, 2506

Ohtaki, Y 2047

Okabe, H 958

Okamoto, T 2232

Okuda, H 1163

Okugawa, Y 2923

Oliveira-Ferrer, L 753

Oliver, SE 1338, 2829

Olmos, D 1655

Olschowski, A 286

Olschwang, S 2728

Olshan, AF 2369

O’Malley Jr, BW 2116

Omlin, A 267

Ong, CJ 399

Ono, H 2765

Ono, M 156

Onuma, H 2765

Oosterom, M 593

Opitz, I 2040

Oppenheim, DE 1221

Oprea, C 2170

Orav, EJ 249

Oriuchi, N 1985, 2030, 2506

Orr, N 1088

Ortega, C 26

Ortiz-Urda, S 1427

Osaka, E 2896

Osanto, S 265

Ostoros, G 55

Osuga, T 156

Oswald, M 976

Ott, K 1712

Ottensmeier, CH 489

Otter, R 850

Otterstrom, C 2040

$\mathrm{Ou}, \mathrm{C} 2300$

Ou, J 1260

Ou, W-B 2479

Overgaard, J 2072

Owczarek, CM 2855

Oyama, T 1985, 2030, 2506

Oza, A 1517

Ozawa, Y 1497

Oze, I 271

Özgüroglu, M 1968, 2996

Öztürk, T 1968, 2996

Paget-Bailly, S 1385

Pagin, M 2865

Paik, JH 2688

Pajares, MJ 1545

Pajic, M 313

Pal, SK 1917

Palani, CD 656

Pallisgaard, N 363

Palmer, CK 1705

Palmqvist, R 2551

Pang, $\mathrm{H} 1351$

Pang, J 1767

Pang, T 916

Pang, B 546

Panteliadou, M 2217

Papadopoulos, A 1385

Parakonthun, T 1712

Pararasa, C 1506

Parfrey, PS 1359

Paris, L 625

Park, CY 278

Park, J 2965

Park, J-Y 34

Park, K 1525

Park, SY 278

Park, W 1420

Park, Y 242, 1328, 2339

Park, YH 384

Parker, C 267

Parmar, D 560
Parmar, M 2178

Passioukov, A 1221

Patard, J-J 1778

Patel, II 2874

Patel, NN 489

Patnick, J 560

Patterson, M 1977

Paul, J 1923

Paulino, V 1307

Pauwels, P 375

Pavlakis, N 2462

Pawłowski, R 991

Payer, F 286

Peake, MD 1936

Pearse, M 2855

Pecqueux, M 44

Peeters, DJE 375

Peeters, M 375

Pelicci, PG 1244

Pelte, M-F 788

Peña-Murillo, C 1221

Penault-Llorca, F 2728

Penfold, MET 1179

Peng, H 1595

Peng, L-X 733

Peng, X 392

Peng, Y 392

Pennati, M 26

Perälä, M 2072

Pereira, SP 1698

Perez-Fernández, T 1334

Perkins, ND 928

Pernemalm, M 2489

Perrone, F 1001

Pestova, E 2985

Peters, GJ 172

Petrachi, T 199

Petrick, JL 2369

Petridis, C 1053

Petty, RD 1525

Pezaro, CJ 267

Pezet, D 2728

Pfannschmidt, J 441

Pfeffer, U 1058

Pheely, A 71

Phelan, CM 530

Phillips, DH 2874

Phillips, N 556

Phull, P 1525

Picardo, E 1133

Piccioli, P 1133

Pichler, J 286

Pichler, M 183, 369, 435, 1614, 2524, 2531

Pierga, JY 2195

Pierotti, MA 1001

Pigozzo, J 1721

Pilling, M 573

Pilotti, S 1001

Pinato, DJ 115, 208, 1930

Pincelli, C 199

Pinchuk, IV 1211

Pinder, SE 1053

Pineda, S 1088

Pinese, M 313

Ping, S 530

Pink, D 1155

Pio, R 1545

Piper, K 489

Pirie, K 2321

Pirrie, SJ 679, 2277

Pisanu, ME 330, 625

Pita, G 1088

Pitkänen, R 2905

Pittet-Cuénod, B 788

Pizzamiglio, S 1001

Pledge, S 1923

Plummer, ER 1977

Plummer, M 7

Pockley, AG 2450

Podo, F 330, 625

Pogue, BW 1698

Pollock, KGJ 2804
Pollock, S 1744

Pontisso, P 2708

Ponzone, R 1133

Poole, C 2369

Pope, JK 2178

Pope, MJ 2178

Porcher, R 1413

Porfiri, E 2821

Porpiglia, M 1133

Porras, I 1139

Port, M 2738

Portillo, I 1334

Posch, C 1427

Potter, J 1891

Potter, VA 908

Potts, A 2804

Pouillon, L 375

Poujol, S 1148

Pouliliou, SE 2217

Powe, DG 1688

Powell, HA 908

Powers, JP 1179

Powles, T 616

Pradere, B 2728

Pretorius, ME 2159

Preusser, M 286

Price, TJ 648

Primrose, J 489

Pritchard, DM 741

Pritz, C 2677

Prizment, AE 2348

Proby C 520

Pruneri, G 1244

Puett, R 1855

Puglisi, F 1133

Pujade-Lauraine, E 1517

Pummer, K 2531

Purdie, CA 565

Purdie, K 520

Pustowka, A 1155

Putter, H 2935

Puviani, M 199

Qian, C-N 733

Qian, H 1199

Qian, J 353, 1169

Qian, X 2662

Qian, Z 1169

Qin, W 189

Qin, Y 2812

Qu, X 1801

Quaglino, P 1721

Quah, C 2756

Quah, TC 1673

Quaife, SL 12

Quarta, S 2708

Queirolo, P 1721

Quinlan, P 565

Quinten, C 2427

Quintero, E 1334

Quirke, P 2178

Qureshi, AA 1855

Radoï, L 1385

Rae, M 1898

Raggi, D 26

Raghavendra, M 146

Rahbari, N 441

Raimond, E 2640

Raimondo, L 2887

Raine, R 1705

Rajan, SS 1744

Rajaraman, P 242

Rajpert-De Meyts, E 668, 2604

Rakha, EA 1688

Ramachandran, R 115

Ramirez, AJ 12

Ramirez Morales, R 2708

Ramos, M 1139

Ramsay, RG 1606

Randall, CJ 489

Ranhem, C 1561

Rani, R 172

Rankin, C 882

Ransohoff, RM 1179

Rappersberger, K 1427

Ratcliffe, J 859

Rau, TT 2985

Ravagnani, F 1001

Real, FX 2123

Recht, L 1179

Redrado, M 764

Rees, RC 2450

Reeve, BB 2427

Reeves, G 224

Reeves, GK 2321

Reichardt, A 1155

Reichardt, P 1155

Reid, G 510

Reid, TR 875

Reig, O 2201

Reimers, MS 459, 2935

Reiner-Concin, A 286

Reissfelder, C 441

Remke, M 636

Ren, C 2291, 2514

Ren, $\mathrm{H} 2812$

Ren, J 1328, 2955

Ren-Heidenreich, L 2812

Reni, M 2165

Repasky, EA 320

Repici, A 1228

Repo, H 2905

Ress, AL 183, 1614

Reulen, RC 2623

Reyal, F 2195

Rha, SY 1433, 1917

Rhodes, JM 741

Ricci, A 330, 625

Richardson, A 1891

Richardson, CA 928

Richman, SD 2178

Ridder, R 1579

Ride, J 1506

Riechelmann, H 2677

Riess, JW 602

Rigas, JR 602 
Roulson, J-A 2099

Rouzaut, A 1545

Rouzier, R 2195

Roxburgh, CSD 1409

Roy, R 2178

Roylance, R 1053

Rubin, G 584

Rubio, G 2700

Rueda, P 764

Ruf, CG 2738

Ruggieri, R 1307

Rugo, HS 2756

Rull, EU 2201

Rümmele, P 2985

Rushton, L 783

Russano, M 2165

Russell, GK 1936

Rustin, GJS 1923

Rustum, YM 1733

Ruszkiewicz, AR 775

Rutgers, EJT 1081

Rutten, A 375

Ruvoletto, M 2708

Ryan, M 560

Ryu, HS 278

Ryu, SY 278

Rzyman, W 991

Saad, RS 609

Saadatmand, S 459

Sabatino, M 2955

Sablin, MP 2195

Sacco, C 26

Saha, MN 2224

Sahdev, A 616

Sahlberg, KK 2072

Sahoo, BS 2144

Saif, MW 1438

Saigusa, S 2923

Saito, R 2047

Saito, S 958

Sakai, D 271, 1163

Sakakura, K 2506

Salas, D 1334

Saltari, A 199

Salvesen, HB 107

Salvesen, ØО 107

Salvioni, R 26

Salvo, E 1545

Samalin, E 1148

Samarasinghe, K 71

Samarzija, M 984

Samatov, T 753

Samonigg, H 183, 435

Sampath, P 1992

Sampson, CM 2178

Sampson, JR 2178

Samra, JS 313

Sanchez, JJ 2662

Sánchez-Carbayo, M 2945

Sánchez-Rovira, P 1139

Sandhu, S 267

Sanfiorenzo, C 1236

Sanghera, B 2847

Sanjmyatav, J 2537

Sanlorenzo, M 1427

Santini, D 2165

Sarasqueta, C 1334

Sarbia, M 2985

Sarker, S-J 266

Saroufim, A 1778

Sasaguri, Y 2583

Sasaki, N 2881

Sasaki, T 1943

Sasazuki, S 1316

Sasieni, P 1841

Sastre, X 2195

Satake, M 1481

Sato, M 469

Sato, T 156

Sato, Y 156, 1497

Satomura, S 2090

Saunders, E 1663
Sava, D 2537

Sava, T 26

Savas, S 1359

Savic, S 2914

Sawada, G 164

Sawada, N 1316

Sawyer, E 1663

Sawyer, EJ 1053

Saxton, JM 831

Saya, H 958

Sayeed, S 1322

Scardino, P 1655

Scarlett, CJ 313

Schaberl-Moser, R 183, 435

Schaeffer, ML 2623

Schall, TJ 1179

Schaller, K 2655

Schartinger, VH 2677

Scheideler, M 1614

Schellens, JHM 2441, 2669

Schenk, P 984

Scheulen, ME 1155

Schinkel, AH 2669

Schmaus, A 1385

Schmelz, H-U 2738

Schmid, D 1862

Schmid, RA 2914

Schmidt, T 441, 1712

Schmoll, H-J 1438

Schmucker von Koch, J 2427

Schnatter, AR 783

Schneider, S 2914

Schneider-Stock, R 2985

Schnüriger, B 1008

Schoemaker, MJ 1898

Schouten, LJ 797

Schouten, PC 2747

Schraml, P 686

Schröder, C 753

Schuler, M 1155

Schumacher, U 753

Schwartz, LH 26

Schwarzenbacher, D 1614

Schweiger, L 1525

Sciarrillo, R 172

Scorfield, S 560

Scorilas, A 1250

Seckl, MJ 1930

Secq, V 1045

Seddon, BM 2420

Seddon, JM 2623

Sedivy, R 286

Seggewies, FS 183

Seigneurin, A 1834

Seitz, JF 1148

Seitz, O 501, 547

Sekino, N 189

Sellars, S 560

Selva, E 1236

Sengupta, D 2144

Sengupta, S 1488

Senter, L 530

Seo, AN 2688

Serdengecti, S 1968, 2996

Serre-Beinier, V 2040

Servetto, A 2887

Seufferlein, T 409

Seymour, MT 2178

Shah, NC 2405

Shah, V 1053

Shahryari, V 1645

Shalaby, T 636

Shamash, J 266

Shao, J-Y 2633

Shapira, I 976

Shapiro, J 648

Sharma, R 115, 208, 1930

Sharp, A 1759

Shaw, GL 2405

Shawyer, A 616

Shelley, CS 146

Shen, D 1169

Shen, D 967
Shen, F 1110

Shen, H 2291

Shen, J 2109, 2662, 2896

Shen, L 1785

Shen, WS 2011

Shen, Z 2021

Shen, Z-B 1552

Sheng, Q 2479

Shi, B 2812

Shi, H 1199

Shi, J 1811

Shi, M 63, 733

Shi, S 2479

Shibuya, K 1316

Shim, S-H 34

Shimada, Y 1571, 2716

Shimazaki, J 2389

Shimazu, T 1316

Shimizu, K 2030, 2047

Shimokawa, T 1163

Shimura, T 2923

Shimwell, NJ 679

Shin, HS 1420

Shin, KH 1420

Shiner, RJ 1930

Shino, M 2506

Shinomiya, I 1053

Shinomiya, N 2881

Shitara, K 271

Sho, M 1027

Sia, KC 94

Sicic, L 1712

Siech, M 409

Siegel, AB 882

Sier, CFM 2935

Siesling, S 850

Siewert, J-R 1712

Sigal-Zafrani, B 2195

Sihto, H 1446

Siler, U 636

Silva, JM 2945

Silverman, DT 2123

Silvestris, N 2165

Simeone, E 1721

Simes, RJ 648

Simmonds, P 230

Simon, AE 12

Simpkins, SA 1744

Simpson, MA 1053

Singer, CF 530

Singh, AP 2000

Singh, K 946

Singh, S 2000

Sini, V 1133

Sinkus, R 1727

Sinyuk, M 1179

Siu, KWM 1250

Siu, LL 648

Sivridis, E 2217

Sjo, OH 2159

Sjöberg, J 2246

Skakkebæk, NE 668

Skrzypski, M 991

Skubitz, KM 304

Skvortsov, S 2677

Skvortsova, I 2677

Slater, J 560

Slavc, I 286

Sleijfer, S 888, 2441

Slotta-Huspenina, J 2081

Smid, K 172

Smit, EF 2427

Smit, JWA 265

Smith, E 775

Smith, JS 2369

Smith, VA 2975

Snijders, PJF 1579

Söderström, M 2905

Soetekouw, PMMB 797

Soh, C-L 2855

Sohda, M 1985

Soltermann, A 2040

Song, C 450, 2472

Song, ES 278

Song, YS 1878

Song, Z 2011

Sonnemann, J 656

Sonoda, L 2847

Soria, D 1688

Southey, MC 1367

Sovio, U 1908

Sowerbutts, AM 573

Spaggiari, L 1244

Spagnolo, F 1721

Sparreboom, A 894

Späth, C 2081, 2544

Speirs, V 1744

Spencer, EA 2321

Spindler, K-LG 363

Spitaleri, G 1244

Spreafico, R 1221

Squiban, D 2655

Squires, J 1359

Srikaran, S 510

Srinivas, S 1917

Srivastava, G 2405

Srivastava, SK 2000

Staehler, M 2821

Stahel, RA 2040

Stallard, N 1950

Stanley, R 1936

Stanley, RA 908

Stanton, L 230

Stapley, S 584

Stavrinos, P 1681

Stebbing, J 4, 1930

Steed, L 831

Steeghs, N 2441

Stefansson, I 107

Stein, MN 2647

Stein, RC 1950

Steinau, H-U 1456

Stenning, S 256

Sternal, M 2537 
Sun, L 695

Sun, W 1438

Sun, X 2369, 2662

Sun, X-M 49

Sun, Y 1169, 1465, 2021, 2283, 2633

Sun, Y-H 1552

Sun, Z 1359

Sunaga, N 2030, 2047

Sundar, S 2837

Sung, F-C 2354

Surrentino, E 625

Suspène, R 2615

Susumu, N 2965

Sutherland, RL 313

Suttle, AB 888, 2647

Sutton, AJ 2837

Suzuki, A 2881

Suzuki, H 2389

Suzuki, K 792

Suzuki, S 1985

Svindland, A 2159

Swanson, J 1402

Swarbrick, A 2462

Swerdlow, AJ 1088, 1898

Swift, RI 19

Swindell, R 1118

Symons, M 1307

Szkandera, J 183, 435

Tabatabai, ZL 1645

Tada, M 1943

Tada, T 2090

Tadler, M 788

Tahahari, D 271

Taiana, E 2865

Taib, NA 2187

Taieb, J 1148

Taipale, J 2246

Tajuddin, SM 2123

Takada, K 156

Takagi, K 1943

Takahara, N 1943

Takahashi, G 2047

Takahashi, H 469

Takahashi, K 2506

Takahashi, M 189

Takahashi, N 2716

Takahashi, Y 164

Takano, M 288

Takano, Y 164

Takashima, A 2716

Takayama, T 1027

Takayasu, Y 2506

Takeda, T 2583

Takeyama, H 469

Takeyoshi, I 2030

Takimoto, R 156

Tamakoshi, A 792

Tamura, F 156

Tan, AM 1673

Tan, AR 2647

Tan, G-H 2187

Tan, H 1748, 2109

Tan, IB 546

Tan, L-D 724

Tan, M-H 1433, 1917

Tan, P 546, 1525

Tan, PS 2708

Tan, TM 115

Tanaka, F 2583

Tanaka, K 2923

Tanaka, T 1027

Tanaka, Y 1645

Tang, D 2593

Tang, G 783

Tang, J 63

Tang, K 2812

Tang, L-L 1465

Tang, W 450

Tang, Z 2021

Tanna, N 4

Tannock, IF 551

Taphoorn, MJB 2427
Tapia, C 2914

Tapper, W 230

Tarazi, J 875, 2821

Tardivon, A 2195

Tardón, A 2123

Tarján, M 1793

Tarragona, J 2544

Tata, LJ 908

Taube, T 2434

Tawadros, T 2099

Taxt, T 107

Taylor, GR 2178

Taylor, MD 636

Taylor, N 1497

Taylor, S 2874

Taylor, SJC 831

Tebbutt, N 648

Tekkis, PP 19

Tekle, C 2072

ten Hoor, KA 1535

Teng, M 2090, 2277

Teng, P-N 123

Teper, E 899

Terrin, L 2708

Terry, KL 1392

Terry, MB 1074, 1367

Testori, A 1721

Thaha, MA 831

Therkildsen, MH 1587

Theunissen, EBM 1081

Thézenas, S 1148

Thibault, F 2195

Thirdborough, SM 489

Thomas, AL 1101

Thomas, BC 2405

Thomas, GJ 489

Thomas, H 1977

Thomas, MC 1705

Thomé, C 286

Thompson, AM 565, 2063

Thompson, PA 214

Thorne, SH 1992

Thurner, E-M 2524

Thygesen, HH 1744

Tian, W 392

Tian, Y-H 297

Tian, Y-M 49, 297

Tibau, A 1139

Tijdens, I 2747

Tille, J-C 788

Tinaglia, V 330

Titmarsh, GJ 2796

Titus, LJ 1392

Tizzoni, L 1244

To, SKY 935

Tod, M 1517

Todd, C 573

Toffalorio, F 1244

Toft, BG 2604

Toiyama, Y 2923

Tominaga, H 1985, 2030, 2506

Tomizawa, Y 2030, 2047

Tomlinson, I 1053

Tonevitsky, A 753

Tong, Z-T 1767

Toraño, EG 2123

Tosi, D 2170

Tot, T 1793

Tóth, K 1733

Touboul, C 2640

Toussi, STK 115

Toyama, S 2389

Toyoda, H 2090

Toyoda, M 2506

Toyozumi, T 189

Tramm, T 2072

Tran, NL 1307

Trembath, RC 1053

Tresca, P 2170

Tretarre, B 1385

Tripathy, D 2756

Triponez, F 2040

Trivier, E 1506
Troppan, K 369

Trovik, J 107

Troy, M 1053

Truini, M 1058

Truzzi, F 199

Tsarouxa, EG 2054

Tseng, D 1179

Tsuburaya, A 546

Tsuda, M 1163

Tsuda, T 271

Tsugane, S 1316, 2765

Tsuji, H 2389

Tsuji, Y 271

Tsujii, H 2389

Tsujinaka, T 1163

Tsujino, T 1943

Tsukamoto, N 1985

Tsushima, Y 1985

Tsuta, K 1571

Tu, D 648

Tucker, MA 2623

Tulin, A 899

Tung, N 530

Tural, D 1968, 2996

Turato, C 2708

Turazza, M 1133

Turna, H 1968, 2996

Turner, EL 2829

Tuynman, J 1606

Twelves, C 1438

Tymrakiewicz, M 1663

Uchi, R 164

Uchida, K 2923

Ueo, H 164

Uesugi, M 1497

Uijterwaal, MH 1579

Ukoumunne, OC 584

Ulcickas Yood, M 2756

Ullah, R 1923

Ulloa, J 1727

Umaña, P 1221

Umesawa, M 792

Umezawa, K 2965

Uramoto, H 2583

Urdinguio, RG 2123

Urquhart, G 1525

Usel, M 788

Utsunomiya, S 271

Uzzo, RG 899

Vaccaro, V 2165

Vahrmeijer, AL 2935

Vahteristo, P 2246

Vaishampayan, UN 1917

Valdimarsdottir, HB 1081

Van Aelst, L 1307

van Altena, AM 42

Van As, N 267

van Dalen, T 1081

van Dam, PA 375

van Dam, P-J 375

van de Laar, R 42

van de Poll-Franse, LV 868

van de Velde, CJH 459, 2935

van de Water, B 2747

van den Brandt, PA 797

van den Elsen, PJ 459

van den Eynden, GGM 375

Van der Gaast, A 2170

van der Graaf, WTA 888

van der Luijt, RB 1081

van der Meer, RW 265

Van der Meulen, IC 593

van der Sanden-Melis, J 108

van Dijk BAC, 850

Van Doorn, L 2170

van Elderen, SGC 265

van Engeland, M 797

Van Gorp, T 42

van Grieken, NCT 546

van Harten, WH 850

Van Hecke, GC 71

van Herpen, CML 888

Van Kemenade, FJ 1579

van Laar, M 1338

van Laere, SJ 375

van Miltenburg, MHAM 2747

van Nimwegen, MJ 2747

van Ooijen, B 1081

van Schinkel, LD 265

van Zandwijk N 510

Vargas, MI 2655

Vartanian, J-P 2615

Vasile, E 2165

Veerapen, N 1506

Vega, R 2700

Veliça, P 1506

Veneziani, BM 2887

Vénissac, N 1236

Vente, JP 1081

Verderio, P 1001

Verdonschot, S 42

Verhoef, S 1081

Verhoest, G 1778

Verkooijen, HM 2187

Vermeulen, PB 375

Verweij, J 888

Veyrat-Follet, C 2170

Viaggi, S 1058

Villeret, J 1045

Vince, GH 286

Vincent, TJ 1402

Vincent-Salomon, A 2195

Vitale, A 2708

Vitonis, AF 1392

Vivaldi, C 2165

Vlastos, G 788

Vleugels, RA 1855

Voest, EE 2441

von Buchwald, C 1587

von Campe, G 286

von Wagner, C 1705

Voss, F 2434

Vowler, SL 2405

Vriens, E 1081

Vrouenraets, BC 1081 
Ward, DG 679, 2277

Ward, LC 546

Ward, MJ 489

Ward, RL 1606

Wardle, J 12

Warot, GA 1269

Warren, AY 2405

Washington, MK 946

Wason, J 1950

Watanabe, M 958

Watanabe, T 1943

Watanabe, Y 792

Waterton, JC 1727

Wattenberg, MM 1472

Weathers, R 2623

Webb, A 489

Weber, DC 2655

Webster, A 55

Weder, W 2040

Wei, B 2011

Wei, J 2662

Wei, J-H 1767

Wei, W 679, 733

Weichert, W 1712

Weihsengruber, F 1427

Weis, J 2427

Weis, S 286

Weiss, C 501, 547

Weitz, J 441

Wejse, PL 1269

Welch, A 1525

Welch, DR 2054

Wen, C 2109

Wenzl, K 369

Werner, HMJ 107

Weston, LL 1211

Wevers, MR 1081

Wheater, M 1759

Whelan, JS 2420

White, J 1744

White, NMA 1250

Whyte, J 976

Wichmann, G 479

Wicklein, D 753

Widhalm, G 286

Wiersma, VR 1535

Wikberg, ML 2551

Wilander, E 1561

Wilbaux, M 1517

Wild, SH 1342

Wilkinson, R 1663

Willemse, PM 265

Willett, WC 249

Williams, BW 304

Williams, GT 2178

Williams, NJ 2829

Wilson, PD 616

Wilson, RH 1923, 2178

Winchester, L 715

Winn, P 1506

Winter, E 1614

Winter, M-P 984

Wiseman, J 1681

Wish, T 1359

Wistuba, II 1545

Witkamp, AJ 1081

Witsch-Baumgartner, M 2677

Witte, BI 1579

Wittekind, C 479

Wittig, S 656

Woehrer, A 286

Womack, C 967

Wong, AST 935
Wong, RPC 399

Wong, RPW 648

Wong, WL 2847

Wongtrakoongate, P 2131

Wood, L 1917

Woods, M 1359

Woolf, DK 2847

Woolhouse, I 1936

Work, ME 1367

Woronoff, A-S 1385

Wu, A 2560

Wu, C-F 341

Wu, G 2250

$\mathrm{Wu}, \mathrm{J} 2109$

Wu, K 249

$\mathrm{Wu}, \mathrm{M} 1110,1811$

Wu, Q 1288, 1958

Wu, R-H 1767

Wu, S 1855

Wu, X 1199

Wu, XB 802

Wu, XS 2011

Wu, Y 1351

Wu, Z 2109

Wunderlich, H 2537

Würtz, F 286

Wuyts, H 375

Wyler, L 686

Wyrwicz, L 991

Wyss, AB 2369

Xi, HQ 2011

Xia, Y 1110

Xiao, C-H 724

Xiao, C-Z 733

Xiao, G 2250

Xiao, Q 1328

Xiao, R 2250

Xiao, W 341

Xiao, W-W 49

Xie, D 1767

Xie, G 2514

Xie, J 2109

Xie, K 2250

Xie, W 842

Xie, X 430

Xiong, S 353

Xu, C-h 808

$\mathrm{Xu}, \mathrm{D} 2496$

Xu, H 916, 1606, 2250

$\mathrm{Xu}, \mathrm{J} 1014,2021,2261,2283,2812$

$\mathrm{Xu}, \mathrm{K} 842$

$\mathrm{Xu}, \mathrm{L} 2109,2261$

Xu, S 1497

$\mathrm{Xu}, \mathrm{W} 1199$

$\mathrm{Xu}, \mathrm{X}$ 63, 392, 817, 1801, 2109

Xu, Y 1169, 2291

Xue, F 1351

Xue, Y 2250

Yagioka, H 1943

Yagiz, K 1269

Yaguchi, T 2965

Yamada, K 1481

Yamada, M 2030, 2047

Yamada, S 2583

Yamada, T 2232

Yamada, Y 2716

Yamaji, T 1316

Yamamoto, H 164

Yamamoto, J 2232

Yamamoto, N 1943

Yamazaki, H 2232
Yan, J 2593

Yan, L 1871

Yan, Y 1606

Yan, Z 1110, 2479

Yang, C-S 2496

Yang, H 2812

Yang, J 2250

Yang, JC-H 2434

Yang, JM 2688

Yang, J-Y 341

Yang, K 2514

Yang, M-H 2354

Yang, P 2896

Yang, Q 1288, 1958

Yang, R-K 2378

Yang, S-N 341

Yang, SR 1359

Yang, T 1199

Yang, X 2896

Yang, Y 1801

Yang, Z 695

Yang, ZH 1655

Yao, Q 2514

Yao, S-Z 1748

Yao, Y 842

Yardley, DA 2756

Yasui, Y 1891

Yasunaga, H 1943

Ychou, M 1148

Ye, C 1066

Ye, L 421

Yeh, K-H 2434

Yen, C-J 2434

Yeoh, AEJ 1673

Yin, X 1169

Yip, C-H 2187

Yokobori, T 1985

Yokoyama, S 2765

Yoo, B 2756

Yoshida, A 1571

Yoshida, M 156

Yoshida, T 1497, 2765

Yoshikawa, T 546

You, B 1517

Young, J 2604

Yousef, GM 1250

Yu, G 2812

Yu, IT-S 2785

Yu, L 2662

Yu, L-G 741

Yu, X-J 2378

Yu, Y 724

Yuan, J 2785

Yuan, Q 2514

Yuan, Y 2514

Yuasa, T 1917

Yue, D 1465

Yue, G 2662

Yuki, S 271

Yun, S 2688

Yusup, G 189

Zablotska, LB 214

Zaffaroni, N 26

Zagonel, V 2165

Zaidi, S 267

Zakikhani, P 1622

Zalcberg, JR 648

Zalewski, P 2821

Zali, N 2708

Zanotti, L 2209

Zanus, G 2708

Zanutto, S 1001
Zapalska, A 2837

Zazo, S 2700

Zeegers, MP 679

Zeestraten, ECM 459, 2935

Zeng, F-f 808

Zeng, J-Z 935

Zeng, L 49, 297

Zeng, M-S 2633

Zeng, W-J 935

Zeng, X-Q 1552

Zettl, A 2544

Zhan, P 967, 1169

Zhan, T 1034

Zhang, B 392, 946, 1199, 1351

Zhang, C 842, 1811, 2560

Zhang, C-x 808

Zhang, D 695, 1767

Zhang, F 1288

Zhang, G 399, 2250

Zhang, H 320, 916, 2021, 2109, 2283

Zhang, J 842, 1169, 2348, 2396

Zhang, J-X 1767

Zhang, L 421, 1199

Zhang, P 1179

Zhang, S 2378

Zhang, SL 546

Zhang, T 1169

Zhang, T-Y 341

Zhang, X 1199, 1801

Zhang, X-Q 2378

Zhang, Y 71, 916, 1260, 1992, 2310

Zhang, Z 63, 2896

Zhao, C 1199

Zhao, H-W 1767

Zhao, J 1359

Zhao, Q 2109

Zhao, X 1260

Zhao, Y 2955

Zheng, J 2116

Zheng, J-N 2496

Zheng, Y 353, 2812

Zhi, L 2583

Zhong, C 733

Zhong, W-M 1767

Zhou, D 2378

Zhou, G-Q 1465, 2633

Zhou, H 2479

Zhou, J 430

Zhou, JY 2310

Zhou, S 409, 2479

Zhou, X 450

Zhu, C 2291

Zhu, J 2300

Zhu, L 1958

Zhu, Q 1199

Zhu, S 450

Zhu, W 1199

Zhu, Y 450, 1359, 2261, 2812

Zhu, Z 2396

Zigeuner, R 2531

Zikos, E 2427

Zlobec, I 1008, 2914

Zmarsly, I 1456

Zou, X 1351, 2250

Zou, X-P 2378

Zou, Y 1992

Zou, Z 2662

Zuber, M 2544

Zupo, S 1058

Zusterzeel, PLM 42 